Pacific Journal of Mathematics

ON THE SOLUTION OF LINEAR G.C.D. EQUATIONS 


\title{
ON THE SOLUTION OF LINEAR G.C.D. EQUATIONS
}

\author{
David Jacobson and Kenneth S. Williams
}

Let $Z$ denote the domain of ordinary integers and let $m(\geqq 1), n(\geqq 1), l_{i}(i=1, \cdots, m), l_{i j}(i=1, \cdots, m ; j=1, \cdots, n) \in Z$. We consider the solutions $x \in Z^{n}$ of

$$
\begin{gathered}
\text { G.C.D. }\left(l_{11} x_{1}+\cdots+l_{1 n} x_{n}+l_{1}, \cdots, l_{m 1} x_{1}+\cdots\right. \\
\left.+l_{m n} x_{n}+l_{m}, c\right)=d,
\end{gathered}
$$

where $c(\neq 0), d(\geqq 1) \in Z$ and G.C.D. denotes "greatest common divisor". Necessary and sufficient conditions for solvability are proved. An integer $t$ is called a solution modulus if whenever $x$ is a solution of (1), $x+t y$ is also a solution of (1) for all $y \in Z^{n}$. The positive generator of the ideal in $Z$ of all such solution moduli is called the minimum modulus of (1). This minimum modulus is calculated and the number of solutions modulo it is derived.

1. Introduction. Let $Z$ denote the domain of ordinary integers and let $m(\geqq 1), n(\geqq 1), l_{i}(i=1, \cdots, m), \quad l_{i j}(i=1, \cdots, m ; j=1, \cdots$, $n) \in Z$. We write $l=\left(l_{1}, \cdots, l_{m}\right)$ and for each $i=1, \cdots, m$ we write $\boldsymbol{l}_{i}=\left(l_{i 1}, \cdots, l_{i n}\right)$ and $\boldsymbol{l}_{i}^{\prime}=\left(l_{i 1}, \cdots, l_{i n}, l_{i}\right)$ so that $\boldsymbol{l} \in Z^{m}$, each $\boldsymbol{l}_{i} \in Z^{n}$, and each $\boldsymbol{l}_{i}^{\prime} \in Z^{n+1}$. If $\boldsymbol{x}=\left(x_{1}, \cdots, x_{n}\right) \in Z^{n}$ we write in the usual way $\boldsymbol{l}_{i} \cdot \boldsymbol{x}$ for the linear expression $l_{i 1} x_{1}+\cdots+l_{i n} x_{n}$. We let $L$ denote the $m \times n$ matrix whose $i$ th row is $l_{i}$ and $L^{\prime}$ denote the $m \times(n+1)$ matrix whose $i$ th row is $\boldsymbol{l}_{i}^{\prime}$.

Henceforth in this paper we will write the abbreviation G.C.D. for "greatest common divisor" of a finite sequence of integers, not all zero, and consider the solutions $\boldsymbol{x} \in Z^{n}$ of

$$
\text { G.C.D. }\left(\boldsymbol{l}_{1} \cdot \boldsymbol{x}+l_{1}, \cdots, \boldsymbol{l}_{m} \cdot \boldsymbol{x}+l_{m}, c\right)=d \text {, }
$$

where $c(\neq 0), d(\geqq 1) \in Z$. A number of authors have either used or proved results concerning special cases of this equation (see for example [1], [5]) so that it is of interest to give a general treatment. This equation is clearly connected with the system

$$
\boldsymbol{l}_{i} \cdot \boldsymbol{x}+l_{i} \equiv 0(\bmod d)(i=1, \cdots, m) \text {. }
$$

If we denote the number of incongruent solutions modulo $d$ of (1.2) by $N\left(d, L^{\prime}\right)$, then $N\left(d, L^{\prime}\right)>0$ is a necessary condition for the solvability of (1.1). A complete treatment of the system (1.2) has been given by Smith [4]. Let $D_{i}=$ greatest common divisor of the determinants of all the $i \times i$ submatrices in $L(i=1, \cdots, \min (m, n)), D_{i}^{\prime}=$ greatest common divisor of the determinants of all the $i \times i$ sub- 
matrices in $L^{\prime}(i=1, \cdots, \min (m, n+1)), \gamma_{i}=$ greatest common divisor of $d$ and $\frac{D_{i}}{D_{i-1}}, i=1, \cdots, \min (m, n)$, where $D_{0}=1$, and $\gamma_{i}^{\prime}=$ greatest common divisor of $d$ and $\frac{D_{i}^{\prime}}{D_{i-1}^{\prime}}, i=1, \cdots, \min (m, n)$, where $D_{0}^{\prime}=1$. Smith has shown that (1.2) is solvable if and only if

$$
\prod_{i=1}^{\min (m, n)} \gamma_{i}=\prod_{i=1}^{\min (m, n)} \gamma_{i}^{\prime}
$$

and

$$
\frac{D_{n+1}^{\prime}}{D_{n}^{\prime}} \equiv 0(\bmod d), \text { if } m>n
$$

When solvable he shows that

$$
N\left(d, L^{\prime}\right)=\gamma d^{\max (n-m, 0)},
$$

where

$$
\gamma=\prod_{i=1}^{\min (m, n)} \gamma_{i} .
$$

We show in Theorem 1 that the conditions

$$
d \mid c, N\left(d, L^{\prime}\right)>0 \text {, G.C.D. }\left(l_{1}, \cdots, l_{m}, d\right)=\text { G.C.D. }\left(l_{1}^{\prime}, \cdots, l_{m}^{\prime}, c\right)
$$

are both necessary and sufficient for solvability of (1.1). When (1.1) is solvable, (1.3) shows that the quantity $g=$ G.C.D. $\left(\boldsymbol{l}_{1}, \cdots, \boldsymbol{l}_{m}, d\right)$ is a factor of $l_{i}, l_{i}(i=1, \cdots, m), c$ and $d$. Cancelling this factor throughout we obtain the equation

$$
\text { G.C.D. }\left(\boldsymbol{l}_{1} / g \cdot \boldsymbol{x}+l_{1} / g, \cdots, \boldsymbol{l}_{m} / g \cdot \boldsymbol{x}+l_{m} / g, c / g\right)=d / g \text {. }
$$

This equation is equivalent to (1.1) in the sense that every solution of this equation is a solution of (1.1) and vice-versa. Thus we can suppose without loss of generality that

$$
\text { G.C.D. }\left(\boldsymbol{l}_{1}, \cdots, \boldsymbol{l}_{m}, d\right)=1 \text {. }
$$

The solution set of (1.1) is denoted by $\mathscr{S}_{d}^{c} \equiv \mathscr{S}_{d}^{c}\left(L^{\prime}\right)$ that is,

$$
\mathscr{S}_{d}^{c} \equiv \mathscr{S}_{d}^{c}\left(L^{\prime}\right)=\left\{\boldsymbol{x} \in Z^{n} \mid \text { G.C.D. }\left(\boldsymbol{l}_{1} \cdot \boldsymbol{x}+l_{1}, \cdots, \boldsymbol{l}_{m} \cdot \boldsymbol{x}+l_{m}, c\right)=d\right\} \text {. }
$$

Moreover when $\mathscr{S}_{d}{ }^{c} \neq \varnothing$, we have

$$
d \mid c, N\left(d, L^{\prime}\right)>0 \text {, G.C.D. }\left(\boldsymbol{l}_{1}^{\prime}, \cdots, \boldsymbol{l}_{m}^{\prime}, c\right)=1,
$$

and we write $e$ for the integer $c / d$.

If $t \in Z, \boldsymbol{a}=\left(a_{1}, \cdots, a_{n}\right) \in Z^{n}$ and $\boldsymbol{b}=\left(b_{1}, \cdots, b_{n}\right) \in Z^{n}$, we say that 
$\boldsymbol{a}$ and $\boldsymbol{b}$ are congruent modulo $t$ (writing $\boldsymbol{a} \equiv \boldsymbol{b}(\bmod t))$ if and only if $a_{i} \equiv b_{i}(\bmod t)$ for each $i=1, \cdots, n$. This congruence $\equiv$ is an equivalence relationship on $Z^{n}$. If $\mathscr{S}_{d}^{c} \neq \varnothing$, any integer $t$ for which this equivalence relationship is preserved on $\mathscr{S}_{d}^{c}\left(\subseteq Z^{n}\right)$ is called a solution modulus of (1.1). Thus a solution modulus $t$ has the property that if $\boldsymbol{x} \in \mathscr{S}_{d}{ }^{c}$ then $\boldsymbol{x}+t \boldsymbol{y} \in \mathscr{S}_{d}{ }^{c}$ for all $\boldsymbol{y} \in Z^{n}$. Clearly 0 and $\pm c$ are solution moduli. In Theorem 2 it is shown that the set of all solution moduli with respect to $\mathscr{S}_{d}^{c}$ viz.,

$$
\mathfrak{M}_{d}^{c} \equiv \mathfrak{M}_{d}^{c}\left(L^{\prime}\right)=\left\{t \in Z \mid \boldsymbol{x}+t \boldsymbol{y} \in \mathscr{S}_{d}^{c} \text { for all } \boldsymbol{x} \in \mathscr{S}_{d}^{c} \text { and all } \boldsymbol{y} \in Z^{n}\right\},
$$

is a principal ideal of $Z$. The positive generator of this ideal is denoted by $M_{d}^{c}\left(L^{\prime}\right)$ and called the minimum modulus of the equation (1.1). We show

$$
M_{d}^{c} \equiv M_{d}^{c}\left(L^{\prime}\right)=d \prod_{p \mid e, N\left(p d, L^{\prime}\right)>0} p .
$$

(Here and throughout this paper the empty product is to be taken as 1). The product in (1.5) is taken over precisely those primes $p \mid e$ for which the system of congruences $\boldsymbol{l}_{i} \cdot \boldsymbol{x}+l_{i} \equiv 0(\bmod p d)(i=1$, $\cdots, m)$ is solvable.

In $\S 5$ we consider the problem of evaluating $\mathfrak{N}_{d}^{c} \equiv \mathfrak{R}_{d}^{c}\left(L^{\prime}\right)$, the number of incongruent solutions $\boldsymbol{x}$ of (1.1) modulo the minimum modulus $M_{d}^{c}$, from which the number of solutions modulo a given modulus can be determined. In Theorem 4 we derive a technical formula which allows the evaluation of $\mathfrak{N}_{d}^{c}$ in some important cases (see $\S 6)$. In particular we prove that if G.C.D. $(d, e)=1$ then

$$
\mathfrak{R}_{d}^{c}=N\left(d, L^{\prime}\right) \prod_{p \mid e, N\left(p d, L^{\prime}\right)>0} p^{n}\left(1-\frac{1}{p^{r(p, L)}}\right),
$$

where $r(p, L)$ is the rank of the matrix $L^{(p)}$ obtained from $L$ by replacing each entry $l_{i j}$ by its residue class modulo $p$ in the finite field $Z_{p}$.

Finally in $\S 7$ an alternative approach is given which enables us to generalize a recent result of Stevens [6].

2. A necessary and sufficient condition for $\mathscr{S}_{d}^{c} \neq \varnothing$. We begin by dealing with the case $d=1$. We prove

LEMMA 1. $\mathscr{S}_{1}^{c} \neq \varnothing$ if and only if

$$
\text { G.C.D. }\left(l_{1}^{\prime}, \cdots, l_{m}^{\prime}, c\right)=1 \text {. }
$$

Proof. The necessity of (2.1) is obvious. Thus to complete the proof it suffices to show that if (2.1) holds then $\mathscr{S}_{1}{ }^{c} \neq \varnothing$. In view of (2.1) for each prime $p \mid c$ there must be some $l_{i}$ or $l_{i j} \equiv \equiv(\bmod p)$. 
If some $l_{i} \not \equiv 0(\bmod p)$ we let $\boldsymbol{x}^{\dagger}(p)=0$, otherwise we have some $l_{i j} \not \equiv$ $0(\bmod p)$ and we let $\boldsymbol{x}^{\dagger}(p)=\left(0, \cdots, 0, x_{j}, 0, \cdots, 0\right)$, where the $j^{\text {th }}$ entry $x_{j}$ is any solution of $l_{i j} x_{j} \equiv 1(\bmod p)$, so that in both cases we have

$$
\text { G.C.D. }\left(\boldsymbol{l}_{1} \cdot \boldsymbol{x}^{\dagger}(p)+l_{1}, \cdots, \boldsymbol{l}_{m} \cdot \boldsymbol{x}^{\dagger}(p)+l_{m}, p\right)=1 \text {. }
$$

We now determine $\boldsymbol{x}$ by the Chinese remainder theorem so that $\boldsymbol{x} \equiv$ $\boldsymbol{x}^{\dagger}(p)(\bmod p)$, for all $p \mid c$. Hence we have

$$
\begin{aligned}
\text { G.C.D. } & \left(\boldsymbol{l}_{1} \cdot \boldsymbol{x}+l_{1}, \cdots, \boldsymbol{l}_{m} \cdot \boldsymbol{x}+l_{m}, \prod_{p \mid c} p\right) \\
= & \prod_{p \mid c} \text { G.C.D. }\left(\boldsymbol{l}_{1} \cdot \boldsymbol{x}+l_{1}, \cdots, \boldsymbol{l}_{m} \cdot \boldsymbol{x}+l_{m}, p\right) \\
= & \prod_{p \mid c}^{\text {G.C.D. }}\left(\boldsymbol{l}_{1} \cdot \boldsymbol{x}^{\dagger}(p)+l_{1}, \cdots, \boldsymbol{l}_{m} \cdot \boldsymbol{x}^{\dagger}(p)+l_{m}, p\right) \\
= & 1,
\end{aligned}
$$

proving that $\boldsymbol{x} \in \mathscr{S}_{1}{ }^{\mathrm{c}}$.

Now we use Lemma 1 to handle the general case $d \geqq 1$. We prove

\section{TheOREM 1. $\mathscr{S}_{d}^{c} \neq \varnothing$ if and only if}

$$
d \mid c, N\left(d, L^{\prime}\right)>0 \text {, G.C.D. }\left(l_{1}, \cdots, l_{m}, d\right)=\text { G.C.D. }\left(l_{1}^{\prime}, \cdots, l_{m}^{\prime}, c\right) \text {. }
$$

Proof. The necessity is obvious. Thus to complete the proof we must show that if (2.2) holds then $\mathscr{S}_{d}{ }^{c} \neq \varnothing$. As $N\left(d, L^{\prime}\right)>0$ there exists $\boldsymbol{k} \in Z^{n}$ and $\boldsymbol{h}=\left(h_{1}, \cdots, h_{m}\right) \in Z^{m}$ such that

$$
\boldsymbol{l}_{i} \cdot \boldsymbol{k}+l_{i}=d h_{i}, i=1, \cdots, m \text {. }
$$

We write $d_{1}=d / g, \boldsymbol{g}_{i}=\boldsymbol{l}_{i} / g \in Z^{n}, \boldsymbol{g}_{i}^{\prime}=\boldsymbol{l}_{i}^{\prime} / g \in Z^{n+1}, g_{i}=l_{i} / g \in Z(i=1, \cdots$, $m$ ) where $g=$ G.C.D. $\left(\boldsymbol{l}_{1}, \cdots, \boldsymbol{l}_{m}, d\right)$ and suppose that

$$
\text { G.C.D. }\left(\boldsymbol{g}_{1}, \cdots, \boldsymbol{g}_{m}, \boldsymbol{h}, e\right)>1 \text {, }
$$

where $e=c / d$. Then there exists a prime $p$ such that

$$
\boldsymbol{g}_{i} \equiv \mathbf{0}(i=1, \cdots, m), \boldsymbol{h} \equiv \mathbf{0}, e \equiv \mathbf{0}(\bmod p) .
$$

Now from (2.3) we have

$$
\boldsymbol{g}_{i} \cdot \boldsymbol{k}+g_{i}=d_{1} h_{i}, i=1, \cdots, m,
$$

and so appealing to $(2.5)$ we deduce $g_{i} \equiv 0(\bmod p)(i=1, \cdots, m)$, giving $\boldsymbol{g}_{i}^{\prime} \equiv \mathbf{0}(\bmod p)(i=1, \cdots, m)$. Thus we have G.C.D. $\left(\boldsymbol{g}_{1}^{\prime}, \cdots\right.$, $\left.\boldsymbol{g}_{m}^{\prime}, d_{1} e\right) \equiv 0(\bmod p)$, which contradicts G.C.D. $\left(\boldsymbol{g}_{1}^{\prime}, \cdots, \boldsymbol{g}_{m}^{\prime}, d_{1} e\right)=1$. Hence our assumption (2.4) is incorrect and we have G.C.D. $\left(g_{1}, \cdots\right.$, $\left.\boldsymbol{g}_{m}, \boldsymbol{h}, e\right)=1$. Thus by Lemma 1 there exists $\lambda \in Z_{n}$ such that

$$
\text { G.C.D. }\left(\boldsymbol{g}_{1} \cdot \boldsymbol{\lambda}+h_{1}, \cdots, \boldsymbol{g}_{m} \cdot \boldsymbol{\lambda}+h_{m}, e\right)=1
$$

and so $\boldsymbol{x}=d_{1} \lambda+\boldsymbol{k} \in \mathscr{S}_{d}^{c}$. 
3. Throughout the rest of this paper we suppose that $\mathscr{S}_{d}{ }^{c} \neq \varnothing$ and G.C.D. $\left(l_{1}, \cdots, l_{m}, d\right)=1$. Thus by Theorem 1 we have $d \mid c, N(d$, $\left.L^{\prime}\right)>0$ and G.C.D. $\left(l_{1}^{\prime}, \cdots, l_{m}^{\prime}, c\right)=1$. Also throughout this paper corresponding to any $\boldsymbol{x} \in \mathscr{S}_{d}^{c}$ we define $\boldsymbol{u} \in Z^{m}$ by $\boldsymbol{u}=\left(u_{1}, \cdots, u_{m}\right)$, where $\boldsymbol{l}_{\boldsymbol{i}} \cdot \boldsymbol{x}+l_{i}=d u_{i}(i=1, \cdots, m)$, so that G.C.D. $(\boldsymbol{u}, e)=1$. The following lemmas will be needed later.

LEMma 2. (i) If $\boldsymbol{x} \in \mathscr{S}_{d}^{\circ}$ and $p$ is a prime dividing e for which the system of simultaneous congruences

$$
l_{i} \cdot z+u_{i} \equiv 0(\bmod p), i=1, \cdots, m,
$$

is solvable then $N\left(p d, L^{\prime}\right)>0$.

(ii) Conversely if $p$ is a prime dividing e for which $N\left(p d, L^{\prime}\right)>0$ then there exists $\boldsymbol{x} \in \mathscr{S}_{d}^{c}$ such that (3.1) is solvable.

Proof. (i) For $\boldsymbol{x} \in \mathscr{S}_{d}^{c}$ and $z$ a solution of (3.1) we let $\boldsymbol{w}=\boldsymbol{x}+d z$. Then for $i=1, \cdots, m$ we have

$$
\begin{aligned}
\boldsymbol{l}_{i} \cdot \boldsymbol{w}+l_{i} & =\left(\boldsymbol{l}_{i} \cdot \boldsymbol{x}+l_{i}\right)+d \boldsymbol{l}_{i} \cdot \boldsymbol{z} \\
& =d\left(u_{i}+\boldsymbol{l}_{i} \cdot \boldsymbol{z}\right) \\
& \equiv 0(\bmod p d),
\end{aligned}
$$

showing that $N\left(p d, L^{\prime}\right)>0$.

(ii) We define $v_{i}$ by $\boldsymbol{l}_{i} \cdot \boldsymbol{w}+l_{i}=p d v_{i}(i=1, \cdots, m)$ and claim that

$$
\text { G.C.D. }\left(\boldsymbol{l}_{1}, \cdots, \boldsymbol{l}_{m}, p v_{1}, \cdots, p v_{m}, e\right)=1 \text {. }
$$

For if not there is a prime $p^{\prime} \mid e$ such that

$$
\boldsymbol{l}_{i} \equiv \mathbf{0}, p v_{i} \equiv 0\left(\bmod p^{\prime}\right)(i=1, \cdots, m) \text {. }
$$

Thus from $l_{i} \cdot \boldsymbol{w}+l_{i}=d p v_{i}$ we have $l_{i} \equiv 0\left(\bmod p^{\prime}\right)(i=1, \cdots, m)$, giving $\boldsymbol{l}_{i}^{\prime} \equiv \mathbf{0}\left(\bmod p^{\prime}\right)(i=1, \cdots, m)$, which contradicts G.C.D. $\left(\boldsymbol{l}_{1}^{\prime}, \cdots\right.$, $\left.\boldsymbol{l}_{m}^{\prime}, \mathrm{de}\right)=1$. Hence (3.2) is valid and so by Lemma 1 we can find $t \in Z^{n}$ such that

$$
\text { G.C.D. }\left(\boldsymbol{l}_{1} \cdot \boldsymbol{t}+p v_{1}, \cdots, \boldsymbol{l}_{m} \cdot \boldsymbol{t}+p v_{m}, e\right)=1 \text {. }
$$

We set $\boldsymbol{x}=\boldsymbol{w}+d \boldsymbol{t}$ so that for $i=1, \cdots, m$ we have

$$
\boldsymbol{l}_{i} \cdot \boldsymbol{x}+l_{i}=d\left(\boldsymbol{l}_{i} \cdot \boldsymbol{t}+p v_{i}\right)
$$

giving

$$
\begin{aligned}
\text { G.C.D. } & \left(\boldsymbol{l}_{1} \cdot \boldsymbol{x}+l_{1}, \cdots, \boldsymbol{l}_{m} \cdot \boldsymbol{x}+l_{m}, c\right) \\
= & d \text { G.C.D. }\left(\boldsymbol{l}_{1} \cdot \boldsymbol{t}+p v_{1}, \cdots, \boldsymbol{l}_{m} \cdot \boldsymbol{t}+p v_{m}, e\right) \\
= & d,
\end{aligned}
$$


so that $\boldsymbol{x} \in \mathscr{S}_{d}{ }^{\circ}$. Finally taking $z=-\boldsymbol{t}$ we see that the system

$$
l_{i} \cdot z+u_{i} \equiv 0(\bmod p)(i=1, \cdots, m)
$$

is solvable, as $u_{i}=l_{i} \cdot t+p v_{i} \cdot$

Lemma 3. Let $t$ be a positive integer, $A$ a subset of $Z^{n}$ which consists of $A(t)$ distinct congruence classes modulo $t$. Now if $t^{\prime}$ is a positive integer such that $t \mid t^{\prime}$ then $A$ consists of $\left(t^{\prime} / t\right)^{n} A(t)$ congruence classes modulo $t^{\prime}$.

Proof. It suffices to prove that a congruence class $C$ modulo $t$ of $A$ consists of $\left(t^{\prime} / t\right)^{n}$ classes modulo $t^{\prime}$. This is clear for if $\boldsymbol{x} \in C$ then so does $\boldsymbol{x}+t \boldsymbol{y}_{i}, \quad\left(i=1, \cdots,\left(t^{\prime} / t\right)^{n}\right)$, where the $\boldsymbol{y}_{\boldsymbol{i}}$ are incongruent modulo $t^{\prime} / t$, moreover the $\boldsymbol{x}+t \boldsymbol{y}_{i}$ are incongruent modulo $t^{\prime}$ and every member of $C$ is congruent modulo $t^{\prime}$ to one of them.

4. The minumum modulus. In this section we determine the minimum modulus $M_{d}^{c}$. We prove

Theorem 2. If $\mathscr{S}_{d}^{c} \neq \varnothing$ and G.C.D. $\left(\boldsymbol{l}_{1}, \cdots, \boldsymbol{l}_{m}, d\right)=1$ the minimum modulus $M_{d}^{c}$ with respect to $\mathscr{S}_{d}^{c}$ is given by

$$
M_{d}^{c}=d \prod_{p \mid e, N\left(p d, L^{\prime}\right)>0} p
$$

Proof. As $\mathscr{S}_{d}^{c} \neq \varnothing, \mathfrak{M}_{d}^{c}$-the set of all solution moduli with respect to $\mathscr{S}_{d}^{c}$-is well-defined and moreover $\mathfrak{M}_{d}^{c}$ is non-empty as 0 and $\pm c$ belong to $\mathfrak{M}_{d}^{c}$. The proof will be accomplished by showing that $\mathfrak{M}_{d}^{c}$ is a principal ideal of $Z$ generated by $d \prod_{p \mid e, N\left(p d, L^{\prime}\right)>0} p$.

(i) We begin by showing that $\mathfrak{M}_{d}^{c}$ is an ideal of $Z$. It suffices to prove that if $t_{1} \in \mathfrak{M}_{d}^{c}$ and $t_{2} \in \mathfrak{M}_{d}^{c}$ then $t_{1}-t_{2} \in \mathfrak{M}_{d}^{c}$. For any $\boldsymbol{x} \in \mathscr{S}_{d}^{c}$ and any $\boldsymbol{y} \in Z^{n}$ we have $\boldsymbol{x}+t_{1} \boldsymbol{y} \in \mathscr{S}_{d}^{c}$, as $t_{1} \in \mathfrak{M}_{d}^{c}$. Hence as $t_{2} \in \mathfrak{M}_{d}^{c}$ we have

$$
\left(\boldsymbol{x}+t_{1} \boldsymbol{y}\right)+t_{2}(-\boldsymbol{y}) \in \mathscr{S}_{d}^{c},
$$

that is

$$
\boldsymbol{x}+\left(t_{1}-t_{2}\right) \boldsymbol{y} \in \mathscr{S}_{d}^{c},
$$

so that

$$
t_{1}-t_{1} \in \mathfrak{M}_{d}^{c} \text {. }
$$

(ii) Next we show that $k=d \prod_{p \mid e, N\left(p d, L^{\prime}\right)>0} p \in \mathfrak{M}_{d}^{c}$. For $\boldsymbol{x} \in \mathscr{S}_{\boldsymbol{d}}^{c}$ and any $\boldsymbol{y} \in Z^{n}$ we have 


$$
\begin{aligned}
\text { G.C.D. } & \left(\boldsymbol{l}_{1} \cdot(\boldsymbol{x}+k \boldsymbol{y})+l_{1}, \cdots, \boldsymbol{l}_{m} \cdot(\boldsymbol{x}+k \boldsymbol{y})+l_{m}, c\right) \\
= & \text { G.C.D. }\left(\boldsymbol{l}_{1} \cdot \boldsymbol{x}+l_{1}+k\left(\boldsymbol{l}_{1} \cdot \boldsymbol{y}\right), \cdots, \boldsymbol{l}_{m} \cdot \boldsymbol{x}+l_{m}+k\left(\boldsymbol{l}_{m} \cdot \boldsymbol{y}\right), d e\right) \\
= & d \text { G.C.D. }\left(u_{1}+k_{1}\left(\boldsymbol{l}_{1} \cdot \boldsymbol{y}\right), \cdots, u_{m}+k_{1}\left(\boldsymbol{l}_{m} \cdot \boldsymbol{y}\right), e\right),
\end{aligned}
$$

where $k_{1}=k / d$. To complete the proof we must show that for all $\boldsymbol{y} \in Z^{n}$ we have

$$
\text { G.C.D. }\left(u_{1}+k_{1}\left(\boldsymbol{l}_{1} \cdot \boldsymbol{y}\right), \cdots, u_{m}+k_{1}\left(\boldsymbol{l}_{m} \cdot \boldsymbol{y}\right), e\right)=1 \text {. }
$$

Suppose that this is not the case. Then there exists $\boldsymbol{y}_{0} \in Z^{n}$ and a prime $p \mid e$ such that $u_{i}+k_{1}\left(\boldsymbol{l}_{i} \cdot \boldsymbol{y}_{0}\right) \equiv 0(\bmod p)$ for $i=1, \cdots, m$. Let $\boldsymbol{z}=\boldsymbol{x}+k \boldsymbol{y}_{0}$ so that for $i=1, \cdots, m$ we have

$$
\begin{aligned}
\boldsymbol{l}_{i} \cdot \boldsymbol{z}+\boldsymbol{l}_{i} & =\boldsymbol{l}_{i} \cdot \boldsymbol{x}+l_{i}+k\left(\boldsymbol{l}_{i} \cdot \boldsymbol{y}_{0}\right) \\
& =d\left(u_{i}+k_{1}\left(\boldsymbol{l}_{i} \cdot \boldsymbol{y}_{0}\right)\right),
\end{aligned}
$$

that is,

$$
\boldsymbol{l}_{i} \cdot \boldsymbol{z}+l_{i} \equiv 0(\bmod p d),
$$

so that $N\left(p d, L^{\prime}\right)>0$. Hence as $p \mid e$ we have $p \mid k_{1}$ and so $p \mid u_{i}$ for $i=1, \cdots, m$. This is the required contradiction as G.C.D. $\left(u_{1}, \cdots\right.$, $\left.u_{m}, e\right)=1$, since $\boldsymbol{x} \in \mathscr{S}_{d}^{c}$.

(iii) In (i) we showed that $\mathfrak{M}_{d}^{c}$ is an ideal of $Z$ and since $Z$ is a principal ideal domain, $\mathfrak{M}_{d}^{c}$ is principal. Thus by the definition of the minimum modulus $M_{d}^{c}$ we have $\mathfrak{M}_{d}^{c}=\left(M_{d}^{c}\right)$. In (ii) we showed that $k \in \mathfrak{M}_{d}^{c}$ so that $M_{d}^{c} \mid k$. Hence to show that $M_{d}^{c}=k$ we have only to show that $k \mid M_{d}^{c}$.

Now for all $\boldsymbol{x} \in \mathscr{S}_{d}^{c}$ and all $\boldsymbol{y} \in Z^{n}$ we have

$$
\text { G.C.D. }\left(\boldsymbol{l}_{1} \cdot\left(\boldsymbol{x}+M_{d}^{\mathrm{c}} \boldsymbol{y}\right)+l_{1}, \cdots, \boldsymbol{l}_{m} \cdot\left(\boldsymbol{x}+M_{d}^{c} \boldsymbol{y}\right)+l_{m}, c\right)=d \text {. }
$$

Hence

$$
\text { G.C.D. }\left(d u_{1}+M_{d}^{c} \boldsymbol{l}_{1} \cdot \boldsymbol{y}, \cdots, d u_{m}+M_{d}^{c} \boldsymbol{l}_{m} \cdot \boldsymbol{y}, d e\right)=d,
$$

and so we must have

$$
M_{d}^{c} \boldsymbol{l}_{i} \cdot \boldsymbol{y} \equiv 0(\bmod d),
$$

for all $\boldsymbol{y} \in Z^{n}$ and all $i(1 \leqq i \leqq m)$. Taking in particular $\boldsymbol{y}=(0, \cdots$, $0,1,0, \cdots, 0)$, where the 1 appears in the $j^{\text {th }}$ place we must have for $i=1, \cdots, m$ and $j=1, \cdots, n$

$$
M_{d}^{c} l_{i j} \equiv 0(\bmod d),
$$

that is

$$
\text { G.C.D. }\left(M_{d}^{c} l_{11}, \cdots, M_{d}^{c} l_{m n}\right) \equiv 0(\bmod d)
$$




$$
\mathrm{M}_{d}^{c} \text { G.C.D. }\left(\boldsymbol{l}_{1}, \cdots, \boldsymbol{l}_{m}\right) \equiv 0(\bmod d) \text {. }
$$

But G.C.D. $\left(\boldsymbol{l}_{1} \cdots, \boldsymbol{l}_{m}, d\right)=1$ so we must have $M_{d}^{c} \equiv 0(\bmod d)$. Thus it suffices to prove that

$$
k_{1} \mid \pi_{d}^{c} \text {, where } k_{1}=k / d=\prod_{p \mid e, N\left(p d, L^{\prime}\right)>0} p \text { and } \pi_{d}^{c}=M_{d}^{c} / d .
$$

We suppose that $k_{1} \nmid \pi_{d}^{c}$ so that there exists a prime $p \mid e$ for which the system $\boldsymbol{l}_{i} \cdot \boldsymbol{w}+l_{i} \equiv 0(\bmod p d)(i=1, \cdots, m)$ is solvable yet $p \nmid$ $\pi_{d}^{c}$. By Lemma 2 (ii) there exists $z \in Z^{n}$ such that for some $\boldsymbol{x} \in \mathscr{S}_{d}^{c}$ we have

$$
l_{i} \cdot z+u_{i} \equiv 0(\bmod p), i=1, \cdots, m .
$$

As $p \nmid \pi_{d}^{c}$ we can define $\lambda$ by $\pi_{d}^{c} \lambda \equiv 1(\bmod p)$ and let $\boldsymbol{y}=\lambda z$ so that for $i=1, \cdots, m$ we have

$$
u_{i}+\pi_{d}^{c} \boldsymbol{l}_{i} \cdot \boldsymbol{y} \equiv 0(\bmod p) .
$$

But as $M_{d}^{c}$ is the minimum modulus and $\boldsymbol{x} \in \mathscr{S}_{d}^{c}$ we must have

$$
\text { G.C.D. }\left(\boldsymbol{l}_{1} \cdot\left(\boldsymbol{x}+M_{d}^{c} \boldsymbol{y}\right)+l_{1}, \cdots, \boldsymbol{l}_{m} \cdot\left(\boldsymbol{x}+M_{d}^{c} \boldsymbol{y}\right)+l_{m}, c\right)=d,
$$

that is

$$
\text { G.C.D. }\left(u_{1}+\pi_{d}^{c} \boldsymbol{l}_{1} \cdot \boldsymbol{y}, \cdots, u_{m}+\pi_{d}^{c} \boldsymbol{l}_{m} \cdot \boldsymbol{y}, e\right)=1 \text {, }
$$

which is contradicted by (4.2). Hence $\pi_{d}^{c}=\prod_{p \mid e, N\left(p d, L^{\prime}\right)>0} p$ and this completes the proof.

We note the following important corollary of Theorem 2 .

CoRollary 1. $\boldsymbol{x} \in Z^{n}$ is a solution of

$$
\text { G.C.D. }\left(\boldsymbol{l}_{1} \cdot \boldsymbol{x}+l_{1}, \cdots, \boldsymbol{l}_{m} \cdot \boldsymbol{x}+l_{m}, c\right)=d
$$

if and only if

$$
\text { G.C.D. }\left(\boldsymbol{l}_{1} \cdot \boldsymbol{x}+l_{1}, \cdots, \boldsymbol{l}_{m} \cdot \boldsymbol{x}+l_{m}, M_{d}^{c}\right)=d .
$$

Proof. (i) Suppose $\boldsymbol{x}$ is a solution of (4.3). Then we can define $u_{i}(i=1, \cdots, m)$ by $\boldsymbol{l}_{i} \cdot \boldsymbol{x}+l_{i}=d u_{i}$ and we have

$$
\text { G.C.D. }\left(u_{1}, \cdots, u_{m}, e\right)=1 \text {. }
$$

Hence we deduce

$$
\text { G.C.D. }\left(u_{1}, \cdots, u_{m}, \prod_{p \mid e, N\left(p d, L^{\prime}\right)>0} p\right)=1
$$


and so

$$
\text { G.C.D. }\left(\boldsymbol{l}_{1} \cdot \boldsymbol{x}+l_{1}, \cdots, \boldsymbol{l}_{m} \cdot \boldsymbol{x}+l_{m}, d \prod_{p \mid e, N\left(p d^{\prime}, L^{\prime}\right)>0} p\right)=d,
$$

which by Theorem 2 is

$$
\text { G.C.D. }\left(\boldsymbol{l}_{1} \cdot \boldsymbol{x}+l_{1}, \cdots, \boldsymbol{l}_{m} \cdot \boldsymbol{x}+l_{m}, M_{d}^{c}\right)=d \text {. }
$$

(ii) Conversely suppose $\boldsymbol{x}$ is a solution of (4.4). Then there exist $u_{i}(i=1, \cdots, m)$ such that $l_{i} \cdot x+l_{i}=d u_{i}$ and

$$
\text { G. C. D. }\left(u_{1}, \cdots, u_{m}, \prod_{p \mid e, N\left(p d, L^{\prime}\right)>0} \mathrm{p}\right)=1 \text {. }
$$

Suppose however that

$$
\text { G.C.D. }\left(u_{1}, \cdots, u_{m}, e\right) \neq 1 \text {. }
$$

Then there exists a prime $p$ such that

$$
u_{i} \equiv 0(i=1, \cdots, m), e \equiv 0(\bmod p), N\left(p d, L^{\prime}\right)=0 .
$$

But for $i=1, \cdots, m$ we have

$$
\boldsymbol{l}_{i} \cdot \boldsymbol{x}+l_{i}=d u_{i} \equiv 0(\bmod p d)
$$

that is $N\left(p d, L^{\prime}\right)>0$, which is the required contradiction. Hence we have

$$
\text { G.C.D. }\left(u_{1}, \cdots, u_{m}, e\right)=1
$$

and so

$$
\text { G.C.D. }\left(\boldsymbol{l}_{1} \cdot \boldsymbol{x}+l_{1}, \cdots, \boldsymbol{l}_{m} \cdot \boldsymbol{x}+l_{m}, c\right)=d \text {. }
$$

5. Number of solutions with respect to the minimum modulus. We begin by evaluating $\mathfrak{N}_{1}^{c}$, that is, the number of solutions of (1.1), when $d=1$, which are incongruent modulo $M_{1}^{c}$. We prove

THEOREM 3. $\quad \mathfrak{I}_{1}^{c}=\prod_{p \mid c, N\left(p, L^{\prime}\right)>0} p^{n}\left(1-\frac{1}{p^{r(p, L)}}\right)$, where $r(p, L)$ is the rank of the matrix $L^{(p)}$ obtained from $L$ by replacing each entry $l_{i j}$ by its residue class modulo $p$ in the finite field $Z_{p}$.

Proof. By Corollary 1 the required number of solutions $\mathfrak{N}_{1}^{c}$ is just the number of solutions taken modulo $M_{1}^{c}$ of

$$
\text { G.C.D. }\left(\boldsymbol{l}_{1} \cdot \boldsymbol{x}+l_{1}, \cdots, \boldsymbol{l}_{m} \cdot \boldsymbol{x}+l_{m}, M_{1}^{c}\right)=1 \text {. }
$$

Thus as $M_{1}^{c}=\prod_{p \mid c, N\left(p, L^{\prime}\right)>0} p$ is a product of distinct primes, a standard 
argument involving use of the Chinese remainder theorem shows that this number $\mathfrak{R}_{1}^{c}$ is just $\prod_{p \mid M_{1}^{c}} \mathfrak{N}(p)$, where $\mathfrak{R}(p)$ is the number of solutions $\boldsymbol{x}$ taken modulo $p$ of

$$
\text { G.C.D. }\left(\boldsymbol{l}_{1} \cdot \boldsymbol{x}+l_{1}, \cdots, \boldsymbol{l}_{m} \cdot \boldsymbol{x}+l_{m}, p\right)=1 \text {. }
$$

Now $\boldsymbol{x}$ is a solution of (5.1) if and only if $\boldsymbol{x}^{(p)}$ is not a solution of the system ( $T$ denotes transpose)

$$
L^{(p)} \boldsymbol{x}^{(p)^{T}}+\boldsymbol{l}^{(p)^{T}}=\mathbf{0}^{T} .
$$

Since $N\left(p, L^{\prime}\right)>0$, this system is consistent over the field $Z_{p}$ and has $p^{n-r(p, L)}$ solutions. Thus the number of solutions (modulo $p$ ) of (5.1) is $p^{n}-p^{n-r(p, L)}=p^{n}\left(1-\frac{1}{p^{r(p, L)}}\right)$, giving

$$
\mathfrak{N}_{1}^{c}=\prod_{p \mid c, N\left(p, L^{\prime}\right)>0} p^{n}\left(1-\frac{1}{p^{r(p, L)}}\right)
$$

as required.

In the proof of Theorem 2 we have seen that any solution modulus $M$ of (1.1) is a multiple of $M_{d}^{c}$. As $\mathscr{S}_{d}^{c}$ consists of $\mathfrak{R}_{d}^{c}$ congruence classes modulo $M_{d}^{c}$, Lemma 3 shows that $\mathscr{S}_{d}^{c}$ consists of $\left(M / M_{d}^{c}\right)^{n} \Re_{d}^{c}$ congruence classes modulo $M$. Hence by Theorem 3 we have

COROLlary 2. The number of solutions $\boldsymbol{x}$ of (1.1), with $d=1$, determined modulo $M-a$ multiple of $M_{d}^{c}-i s$

$$
M^{n} \prod_{p \mid c, N\left(p, L^{\prime}\right)>0}\left(1-\frac{1}{p^{r(p, L)}}\right) .
$$

As a consequence of Corollary 2 we have the linear case of a result recently established by Stevens [6]. A generalization of this result is proved in $\S 7$.

Corollary 3. (Stevens) The number of solutions of

$$
\text { G.C.D. }\left(a_{1} x_{1}+b_{1}, \cdots, a_{n} x_{n}+b_{n}, c\right)=1 \text {, }
$$

taken modulo $c$, is

$$
c^{n} \prod_{p \mid c}\left(1-\frac{\nu_{1}(p) \cdots \nu_{n}(p)}{p^{n}}\right),
$$

where $\nu_{i}(p)(i=1, \cdots, n)$ is the number of incongruent solutions modulo $p$ of $a_{i} x_{i}+b_{i} \equiv 0(\bmod p)$.

Proof. The system 


$$
a_{i} x_{i}+b_{i} \equiv 0(\bmod p)(i=1, \cdots, n),
$$

is solvable if and only if

$$
\text { G.C.D. }\left(a_{i}, p\right) \mid b_{i}(i=1, \cdots, n) \text {, }
$$

that is, if and only if

$$
p \nmid a_{i} \text { or } p \mid \text { G.C.D. }\left(a_{i}, b_{i}\right)(i=1, \cdots, n) \text {. }
$$

Hence by Corollary 2 the required number of solutions is

$$
c^{n} \prod_{p \mid c}^{\prime}\left(1-\frac{1}{p^{r(p)}}\right),
$$

where the dash (') denotes that the product is taken over all $p$ such that $p \nmid a_{i}$ or $p \mid$ G.C.D. $\left(a_{i}, b_{i}\right)(1 \leqq i \leqq n)$ and $r(p)$ is the number of $a_{i}(i=1, \cdots, n)$ not divisible by $p$. As

$$
\nu_{i}(p)=\left\{\begin{array}{l}
1, p \nmid a_{i}, \\
0, p \mid a_{i}, p \nmid b_{i}, \\
p, p\left|a_{i}, p\right| b_{i},
\end{array}\right.
$$

for $i=1, \cdots, n,(5.2)$ is just

$$
c^{n} \prod_{p \mid c}\left(1-\frac{\nu_{1}(p) \cdots \nu_{n}(p)}{p^{n}}\right),
$$

which is the required result.

We now turn to the general case $d \geqq 1$. Let $p$ be a prime and let $E$ denote an equivalence class of $\mathscr{S}_{d}^{c}$ consisting of elements of $\mathscr{S}_{d}^{c}$ which are congruent modulo $d$. We assert that if $\boldsymbol{x}^{(1)}, \boldsymbol{x}^{(2)} \in E$ then the system $l_{i} \cdot z^{(1)}+u_{i}^{(1)} \equiv 0(\bmod p)(i=1, \cdots, n)$ is solvable if and only if the system $\boldsymbol{l}_{i} \cdot \boldsymbol{z}^{(2)}+u_{i}^{(2)} \equiv 0(\bmod p)(i=1, \cdots, n)$ is solvable. As $\boldsymbol{x}^{(1)} \equiv \boldsymbol{x}^{(2)}(\bmod p)$ there exists $\boldsymbol{t} \in Z^{n}$ such that $\boldsymbol{x}^{(2)}=\boldsymbol{x}^{(1)}+d \boldsymbol{t}$. Hence for $i=1, \cdots, n$ we have

$$
\begin{aligned}
d u_{i}^{(2)} & =\boldsymbol{l}_{i} \cdot \boldsymbol{x}^{(2)}+l_{i} \\
& =\boldsymbol{l}_{i} \cdot \boldsymbol{x}^{(1)}+l_{i}+d \boldsymbol{l}_{i} \cdot \boldsymbol{t} \\
& =d u_{i}^{(1)}+d \boldsymbol{l}_{i} \cdot \boldsymbol{t}
\end{aligned}
$$

giving

$$
u_{i}^{(2)}=u_{i}^{(1)}+\boldsymbol{l}_{i} \cdot \boldsymbol{t}
$$

If there exists $\boldsymbol{z}^{(1)} \in Z^{n}$ such that $\boldsymbol{l}_{i} \cdot \boldsymbol{z}^{(1)}+u_{i}^{(1)} \equiv 0(\bmod p)(i=1$, $\cdots, n$ ) letting $\boldsymbol{z}^{(2)}=\boldsymbol{z}^{(1)}-\boldsymbol{t}$ we have $\boldsymbol{l}_{i} \cdot \boldsymbol{z}^{(2)}+u_{i}^{(2)}=\boldsymbol{l}_{i} \cdot \boldsymbol{z}^{(1)}-\boldsymbol{l}_{i} \cdot \boldsymbol{t}+u_{i}^{(1)}+$ $\boldsymbol{l}_{\boldsymbol{i}} \cdot \boldsymbol{t} \equiv 0(\bmod p)$, which completes the proof of the assertion. Hence 
the solvability of the system

$$
\boldsymbol{l}_{i} \cdot z+u_{i} \equiv 0(\bmod p)(i=1, \cdots, n)
$$

depends only on the equivalence class $E$ to which $\boldsymbol{x}$ (recall $\boldsymbol{l}_{i} \cdot \boldsymbol{x}+l_{i}=$ $d u_{i}$ ) belongs. Thus we can define a symbol $\delta_{p}(E)$ as follows:

$$
\delta_{p}(E)=\left\{\begin{array}{l}
1, \text { if for some } \boldsymbol{x} \in E(\text { and thus for all } \boldsymbol{x} \in E) \text { the system } \\
\boldsymbol{l}_{i} \cdot z+u_{i} \equiv 0(\bmod p)(i=1, \cdots, m) \text { is solvable, } \\
0, \text { otherwise. }
\end{array}\right.
$$

We now prove the following result.

THEOREM 4. $\mathfrak{N}_{d}^{c}=\sum_{j=1}^{N\left(d, L^{\prime}\right)}\left\{\prod_{p \mid e, N\left(p d, L^{\prime}\right)>0} p^{n}\left(1-\frac{1}{p^{r(p, L)}}\right)^{\delta_{p}\left(E^{(j)}\right)}\right\}$, where the $E^{(j)}$ denote the $N\left(d, L^{\prime}\right)$ congruence classes modulo $d$ in $\mathscr{S}_{d}{ }^{c}$.

Proof. We let

$$
\mathscr{S}=\left\{\boldsymbol{x} \in Z^{n} \mid \boldsymbol{l}_{i} \cdot \boldsymbol{x}+l_{i} \equiv 0(\bmod d), i=1, \cdots, m\right\}
$$

so that we have $\mathscr{S}_{d}^{c} \subseteq \mathscr{S}$. Now $\mathscr{S}$ consists of $N\left(d, L^{\prime}\right)$ congruence classes modulo $d$ and if we restrict this equivalence relation modulo $d$ to $\mathscr{S}_{d}^{c}$, we show that $\mathscr{S}_{d}^{c}$ also contains the same number of classes. We write $E(\boldsymbol{x})\left(\operatorname{resp} . E^{\prime}(\boldsymbol{x})\right)$ for the equivalence class to which $\boldsymbol{x} \in \mathscr{S}_{d}{ }^{c}$ (resp. $x \in \mathscr{S}$ ) belongs. From the proof of Theorem 1 we see that for each $\boldsymbol{x} \in \mathscr{S}$ there exists $\lambda \in Z^{n}$ such that $\boldsymbol{x}+d \lambda \in \mathscr{S}_{d}^{c}$. We define a mapping $f$ from the set of equivalence classes of $\mathscr{S}$ into the set of equivalence classes of $\mathscr{S}_{d}^{c}$ as follows: For $\boldsymbol{x} \in \mathscr{S}$

$$
f\left(E^{\prime}(\boldsymbol{x})\right)=E(\boldsymbol{x}+d \lambda) \text {. }
$$

This mapping is well-defined for if $\boldsymbol{x}^{\prime} \in \mathscr{S}$ is such that $E^{\prime}\left(\boldsymbol{x}^{\prime}\right)=E^{\prime}(\boldsymbol{x})$ then $E\left(\boldsymbol{x}^{\prime}+d \lambda^{\prime}\right)=E(\boldsymbol{x}+d \lambda) . f$ is onto for if $\boldsymbol{x} \in \mathscr{S}_{d}{ }^{c}$ then $f\left(E^{\prime}(\boldsymbol{x})\right)=$ $E(\boldsymbol{x})$ and is also one-to-one, for if $f\left(E^{\prime}(\boldsymbol{x})\right)=f\left(E^{\prime}(\boldsymbol{y})\right)$, then $E(\boldsymbol{x}+d \boldsymbol{\lambda})=$ $E\left(\boldsymbol{y}+d \lambda^{\prime}\right)$, that is $\boldsymbol{x} \equiv \boldsymbol{y}(\bmod d)$, giving $E^{\prime}(\boldsymbol{x})=E^{\prime}(\boldsymbol{y})$. Thus the number of equivalence classes of $\mathscr{S}_{d}^{c}$ is the same as the number of equivalence classes of $\mathscr{S}$, that is $N\left(d, L^{\prime}\right)$.

Since $d \mid M_{d}^{c}$, each equivalence class $E$ of $\mathscr{S}_{d}^{c}$, consists of a certain number of distinct classes in $\mathscr{S}_{d}^{c}$ modulo $M_{d}^{c}$. We now determine this number. If $\boldsymbol{x} \in E, \boldsymbol{x}+d \boldsymbol{t}$ also belongs in $E$ if and only if it belongs in $\mathscr{S}_{d}{ }^{c}$, that is, if and only if,

$$
\text { G.C.D. }\left(\boldsymbol{l}_{1} \cdot(\boldsymbol{x}+d \boldsymbol{t})+l_{1}, \cdots, \boldsymbol{l}_{m} \cdot(\boldsymbol{x}+d \boldsymbol{t})+l_{m}, c\right)=d,
$$

that is, if and only if, 


$$
\text { G.C.D. }\left(u_{1}+l_{1} \cdot t, \cdots, u_{m}+l_{m} \cdot t, e\right)=1 \text {. }
$$

Thus the number of distinct classes modulo $M_{d}^{c}$ contained in $E$ is just the number of distinct classes modulo $\pi_{d}^{c}=M_{d}^{c} / d$ which satisfy (5.3). But the minimum modulus of (5.3) is $\Pi_{p l e} p^{\delta} p^{(E)}$. By lemma 2 (i) $\delta_{p}(E)=1$ implies $N\left(p d, L^{\prime}\right)>0$, so that $\prod_{p \mid e} p^{\delta} p^{(E)}$ divides $\Pi_{p \mid e, N\left(p d, L^{\prime}\right)>0} \quad p=\pi_{d}^{c}$. Writing $\Pi_{p \mid e}^{+}$for $\Pi_{p \mid e, N\left(p d, L^{\prime}\right)>0}$ and $\Pi_{p \mid e}^{0}$ for $\Pi_{p \mid e, N\left(p d, L^{\prime}\right)=0}$, the required number of classes is by Corollary 2

$$
\begin{aligned}
& =\prod_{p \mid e}^{+} p^{n} \cdot \prod_{p \mid e}\left(1-\frac{1}{p^{r(p, L)}}\right)^{\delta_{p}(E)} \\
& =\prod_{p \mid e}^{+} p^{n}\left(1-\frac{1}{p^{r(p, L)}}\right)^{\delta_{p}(E)} \cdot \quad \prod_{p \mid e}^{0}\left(1-\frac{1}{p^{r(p, L)}}\right)^{\delta_{p}(E)} \\
& =\prod_{p \mid e}^{+} p^{n}\left(1-\frac{1}{p^{r(p, L)}}\right)^{\delta_{p}(E)},
\end{aligned}
$$

as $N\left(p d, L^{\prime}\right)=0$ implies $\delta_{p}(E)=0$.

Finally letting $E^{(1)}, \cdots, E^{(h)}$ denote the $h=N\left(d, L^{\prime}\right)$ distinct equivalence classes in $\mathscr{S}_{d}^{c}$ we deduce that the total number of incongruent solutions modulo $M_{d}^{c}$ of (1.1) is

$$
\sum_{\jmath=1}^{N\left(\lambda, L^{\prime}\right)}\left\{\prod_{p \mid e, N\left(p d, L^{\prime}\right)>0} p^{n}\left(1-\frac{1}{p^{r(p, L)}}\right)^{\delta_{p}\left(E^{(\jmath)}\right)}\right\}
$$

We remark that $r(p, L) \neq 0$, for $p \mid e$ and $\delta_{p}(E)=1$. Otherwise, if $r(p, L)=0, \boldsymbol{l}_{i} \equiv \mathbf{0}(\bmod p)(i=1, \cdots, m)$. But as $\delta_{p}(E)=1$ then for $\boldsymbol{x} \in E$ the system $l_{i} \cdot z+u_{i} \equiv 0(\bmod p)(i=1, \cdots, m)$ is solvable contradicting G.C.D. $\left(u_{1}, \cdots, u_{m}, e\right)=1$.

6. Some special cases. We note a number of interesting cases of our results.

Corollary 4. If G.C.D. $(d, e)=1$ then the number $\mathfrak{R}_{d}^{c}$ of solutions of (1.1) modulo $M_{d}^{c}$ is

$$
\mathfrak{N}_{d}^{c}=N\left(d, L^{\prime}\right) \prod_{p\left\lfloor e, N\left(p d, L^{\prime}\right)>0\right.} p^{n}\left(1-\frac{1}{p^{r(p, L)}}\right) .
$$

Proof. By Theorem 4 it suffices to show that if G.C.D. $(d, e)=$ $1, p \mid e, N\left(p d, L^{\prime}\right)>0$ then for all $\boldsymbol{x} \in \mathscr{S}_{d}^{c}$ we have $\delta_{p}(E)=1$, that is the system $\boldsymbol{l}_{i} \cdot \boldsymbol{z}+u_{i} \equiv 0(\bmod p)$ is solvable. Let $\boldsymbol{w}$ be a solution of $\boldsymbol{l}_{i} \cdot \boldsymbol{w}+l_{i} \equiv 0(\bmod p d)$, say $\boldsymbol{l}_{i} \cdot \boldsymbol{w}+l_{i}=p d v_{i}(i=1, \cdots, m)$. As $p \nmid d$ we can define $z=d^{-1}(\boldsymbol{w}-\boldsymbol{x})$, where $d d^{-1} \equiv 1(\bmod p)$ so that for $i=$ $1, \cdots, m$ we have 


$$
\begin{aligned}
\boldsymbol{l}_{\boldsymbol{i}} \cdot \boldsymbol{z}+u_{i} & =d^{-1}\left(\boldsymbol{l}_{i} \cdot \boldsymbol{w}-\boldsymbol{l}_{i} \cdot \boldsymbol{x}\right)+u_{i} \\
& =d^{-1}\left(p d v_{i}-l_{i}-d u_{i}+l_{i}\right)+u_{i} \\
& =d d^{-1}\left(p v_{i}-u_{i}\right)+u_{i} \\
& \equiv 0(\bmod p),
\end{aligned}
$$

as required.

CoROLlaRY 5. If $N\left(d, L^{\prime}\right)=1$ then the number $\mathfrak{N}_{d}^{c}$ of solutions of (1.1) modulo $M_{d}^{c}$ is

$$
\mathfrak{R}_{d}^{c}=\prod_{p \mid e, N\left(p d, L^{\prime}\right)>0} p^{n}\left(1-\frac{1}{p^{r(p, L)}}\right) .
$$

In particular $N\left(d, L^{\prime}\right)=1$ when $L$ is invertible $(\bmod d)$, and so $\mathfrak{N}_{d}^{c}$ is given by (6.1). Moreover if $L$ is invertible modulo $d \prod_{p l e} p$ or $c$, then (1.1) is solvable and $\mathfrak{N}_{d}^{c}=\Pi_{p \mid e}\left(p^{n}-1\right)$.

Proof. This is immediate from Theorem 4 since by Lemma 2(ii), $\delta_{p}(E)=1$ for all $p \mid e, N\left(p d, L^{\prime}\right)>0$. Also (1.1) is solvable when $L$ is invertible modulo $d \prod_{p \mid e} p$ as

$$
\text { G.C.D. }\left(\boldsymbol{l}_{1}, \cdots, \boldsymbol{l}_{m}, d\right)=\text { G.C.D. }\left(\boldsymbol{l}_{1}^{\prime}, \cdots, \boldsymbol{l}_{m}^{\prime}, c\right)=1 \text {. }
$$

CoROLlaRY 6. If $L$ is invertible modulo $\prod_{p \mid e, N\left(p d, L^{\prime}\right)>0} p$ then the number of solutious of (1.1) modulo $M_{d}^{c}$ is

$$
\mathfrak{N}_{d}^{c}=N\left(d, L^{\prime}\right) \prod_{p \mid e, N\left(p d, L^{\prime}\right)>0}\left(p^{n}-1\right) \text {. }
$$

Proof. Let $p$ be any prime such that $p \mid e$ and $N\left(p d, \mathrm{~L}^{\prime}\right)>0$. Then $L$ is invertible modulo $p$ and so for any $\boldsymbol{x} \in \mathscr{S}_{d}^{c}$ the system

$$
\boldsymbol{l}_{i} \cdot z+u_{i} \equiv 0(\bmod p)(1=1, \cdots, n)
$$

is solvable and so $\delta_{p}\left(E^{(j)}\right)=1, \mathrm{j}=1, \cdots, N\left(d, L^{\prime}\right)$. Moreover as $L$ is invertible modulo $p$ we have $r(p, L)=n$ and the result follows from Theorem 4.

CoRollary 7. If

$$
\text { G.C.D. }\left(a_{1}, \cdots, a_{n}, d\right)=1
$$

the equation

$$
\text { G.C.D. }\left(a_{1} x_{1}+\cdots+a_{n} x_{n}+b, c\right)=d
$$

is solvable if and only if

$$
d \mid c, \text { G.C.D. }\left(a_{1}, \cdots, a_{n}, b, c\right)=1 \text {. }
$$


The minimum modulus of (6.3) is

$$
d \prod_{p \mid c / d}^{\prime} p
$$

and the number of solutions $\boldsymbol{x}$ modulo this minimum modulus is

$$
d^{n-1} \prod_{p \mid c / d}^{\prime}\left(p^{n}-p^{n-1}\right),
$$

where the dash (') means that the product is taken over those primes $p \mid c / d$ such that G.C.D. $\left(a_{1}, \cdots, a_{n}, p\right)=1$.

Proof. According to Smith [4] or Lehmer [3] the number of solutions $\boldsymbol{x}$ taken modulo $d$ of

$$
a_{1} x_{1}+\cdots+a_{n} x_{n}+b \equiv 0(\bmod d)
$$

is $d^{n-1}$ G.C.D. $\left(a_{1}, \cdots, a_{n}, d\right)$ if G.C.D. $\left(a_{1}, \cdots, a_{n}, d\right)$ divides $b$ and 0 otherwise. Thus as G.C.D. $\left(a_{1}, \cdots, a_{n}, d\right)=1$, we have $N\left(d, L^{\prime}\right)=d^{n-1}$ and so by Theorem $1(6.3)$ is solvable if and only if

$$
d \mid c \text {, G.C.D. }\left(a_{1}, \cdots, a_{n}, b, c\right)=1 \text {. }
$$

Now if (6.3) is solvable and $p \mid c / d$ then

$$
\text { G.C.D. }\left(a_{1}, \cdots, a_{n}, p d\right) \mid b
$$

if and only if

$$
\text { G.C.D. }\left(a_{1}, \cdots, a_{n}, p\right)=1 \text {, }
$$

in view of (6.2) and (6.4). Thus by Theorem 2 the minimum modulus is

$$
d \prod_{p|c| d}^{\prime} p
$$

Finally for $p \mid c / d$, G.C.D. $\left(a_{1}, \cdots, a_{n}, p\right)=1$ we have $r(p, L)=1$ and moreover the congruence $a_{1} x_{1}+\cdots+a_{n} x_{n}+u \equiv 0(\bmod p)$ is always solvable so that $\delta_{p}\left(E^{(j)}\right)=1, j=1, \cdots, d^{n-1}$. Hence by Theorem 4 the number of solutions is

$$
d^{n-1} \prod_{p \mid c / d}^{\prime} p^{n}\left(1-\frac{1}{p}\right) .
$$

We remark that in particular ([5])

$$
\text { G.C.D. }(a x+b, c)=1
$$

is solvable if and only if G.C.D. $(a, b, c)=1$, has minimum modulus $\Pi_{p \mid c, p \nmid a} p$, and has $\Pi_{p \mid c, p \nmid a}(p-1)$ solutions $x$ modulo the minimum modulus. 
COROLlaRY 8. There is a unique solution of (1.1) modulo $M_{d}^{c}$ if and only if

(i) $N\left(d, L^{\prime}\right)=1$ and there is no prime $p$ such that

$$
p \mid e, N\left(p d, L^{\prime}\right)>0,
$$

or

(ii) $N\left(d, L^{\prime}\right)=1$ and the only prime $p$ such that $p \mid e, N\left(p d, L^{\prime}\right)>$ 0 , is $p=2$, and $r(2, L)=1, n=1$.

Proof. If (1.1) possesses a unique solution modulo $M_{d}^{c}$, Theorem 4 shows that $S$ can consist only of a single congruence class modulo d. Hence $N\left(d, L^{\prime}\right)=1$. Also by Theorem 4 if there is no prime $p$ such that $p \mid e$ and $N\left(p d, L^{\prime}\right)>0$ then $\Re_{d}^{c}=1$. Suppose however that there is such a prime $p$. Then by Corollary 5 we have

$$
1=\prod_{p \mid e, N\left(p d, L^{\prime}\right)>0}\left(p^{n}-p^{n-r(p, L)}\right) .
$$

This occurs if and only if

$$
p^{n}-p^{n-r(p, L)}=1,
$$

for all $p \mid e$ with $N\left(p d, L^{\prime}\right)>0$. But the left-hand side of (6.5) is divisible by $p$ unless $r(p, L)=n$. Then $p^{n}=2$ and we have $p=2$, $n=1, r(p, L)=r(2, L)=1$, which proves the theorem.

7. Another method. Although the formula of Theorem 4 applies to some important cases in $\S 6$, this formula seems difficult to evaluate even for example in the diagonal case

$$
\text { G.C.D. }\left(a_{1} x_{1}+b_{1}, \cdots, a_{n} x_{n}+b_{n}, c\right)=d \text {. }
$$

The inherent difficulty is in determining for a given prime $p$ which solutions of this equation have the property that the system $a_{i} z_{i}+$ $u_{i} \equiv 0(\bmod p)(i=1, \cdots, n)$ is solvable. We now present another method which in conjunction with previous results yields the diagonal case.

We consider the set $\mathfrak{U}$ of $\boldsymbol{u} \in Z^{m}$ with G.C.D. $(\boldsymbol{u}, e)=1$ for which the system

$$
\boldsymbol{l}_{i} \cdot \boldsymbol{x}+l_{i} \equiv d u_{i}(\bmod c)(i=1, \cdots, n) \text { is solvable. }
$$

It is clear that if $\boldsymbol{u} \in \mathfrak{U}$ and $\boldsymbol{u} \equiv \boldsymbol{u}^{\prime}(\bmod e)$ then $\boldsymbol{u}^{\prime} \in \mathfrak{U}$. We denote by $K_{d}^{c}$ the number of distinct classes modulo $e$ contained in $\mathfrak{u}$. Let $\mathfrak{N}$ denote the number of solutions $\boldsymbol{x}$ of (1.1) modulo $c$. We prove

THeOREM 5. $\mathfrak{N}=K_{d}^{c} N_{c}\left(L^{*}\right)$ where $L^{*}$ is the $m \times(n+1)$ matrix 
$[L: 0]$.

Proof. If $\boldsymbol{x} \in \mathscr{S}_{d}^{c}$ then there exists $\boldsymbol{u} \in Z^{n}$ such that $\boldsymbol{l}_{\boldsymbol{i}} \cdot \boldsymbol{x}+l_{\boldsymbol{i}}=$ $d u_{i}(i=1, \cdots, m)$ and G.C.D. $(\boldsymbol{u}, e)=1$. If $\boldsymbol{x}, \boldsymbol{x}^{\prime} \in \mathscr{S}_{d}^{c}$ are such that

$\boldsymbol{x} \equiv \boldsymbol{x}^{\prime}(\bmod e)$ then $d u_{i} \equiv d u_{i}^{\prime}(\bmod c)$, that is $u_{i} \equiv u_{i}^{\prime}(\bmod e)$.

Conversely if G.C.D. $(\boldsymbol{u}, e)=1$ and $\boldsymbol{x}$ satisfies $\boldsymbol{l}_{i} \cdot \boldsymbol{x}+l_{i} \equiv d u_{i}(\bmod$ c) $(i=1, \cdots, m)$ then $\boldsymbol{l}_{i} \cdot \boldsymbol{x}+l_{i}=d\left(u_{i}+\lambda_{i} e\right)$ and $\boldsymbol{x} \in \mathscr{S}_{d}^{c}$ as G.C.D. $(\boldsymbol{u}+$ $\lambda e, e)=$ G.C.D. $(\boldsymbol{u}, e)=1$.

Thus $\boldsymbol{x} \in \mathscr{S}_{d}^{c}$ if and only if $\boldsymbol{x}$ is a solution of $\boldsymbol{l}_{i} \cdot \boldsymbol{x}+l_{i} \equiv d u_{i}(\bmod$ $c)$, where G.C.D. $(\boldsymbol{u}, e)=1$. Now there are $K_{d}^{c}$ incongruent classes of $\boldsymbol{u}$ modulo $e$, with G.C.D. $(\boldsymbol{u}, e)=1$, for which (7.1) is solvable. For each one of these, (7.1) has $N_{c}(L: 0)$ incongruent solutions modulo $c$. Hence we have

$$
\mathfrak{R}=K_{d}^{c} N_{c}\left(L^{*}\right)
$$

as required.

We now obtain the following interesting result.

CoROLlary 9. If $\boldsymbol{h} \in Z^{n}$ and $e_{1}, \cdots, e_{n}$ are divisors of $e$ then the system

$$
u_{i} \equiv h_{i}\left(\bmod e_{i}\right)(i=1, \cdots, n)
$$

has a solution $\boldsymbol{u}=\left(u_{1}, \cdots, u_{n}\right)$ such that G.C.D. $(\boldsymbol{u}, e)=1$ if and only if G.C.D. $\left(e_{1}, \cdots, e_{n}, h_{1}, \cdots, h_{n}, e\right)=1$. When this holds (7.2) has

$$
\prod_{i=1}^{n}\left(e / e_{i}\right) \prod_{p \mid e}^{\prime}\left(1-\frac{1}{p^{r(p)}}\right)
$$

distinct solutions $\boldsymbol{u}$ modulo e, for which G.C.D. $(\boldsymbol{u}, e)=1$, where $r(p)=$ number of $e_{i}(i=1, \cdots, n)$ not divisible by $p$, and the dash (') means that the product is taken over those primes $p \mid e$ such that $p \nmid e_{i}$ or $p \mid$ G.C.D. $\left(e_{i}, h_{i}\right)(i=1, \cdots, n)$.

Proof. The system (7.2) has a solution $\boldsymbol{u}$ such that G.C.D. $(\boldsymbol{u}, e)=1$ if and only if

$$
\text { G.C.D. }\left(e_{1} x_{1}+h_{1}, \cdots, e_{n} x_{n}+h_{n}, e\right)=1
$$

is solvable, which by Lemma 1 is the case if and only if G.C.D. $\left(e_{1}\right.$, $\left.\cdots, e_{n}, h_{1}, \cdots, h_{n}, e\right)=1$. Applying Theorem 5 to $(7.3)$ we have $\mathfrak{N}=$ $K_{1}^{e} N_{e}\left(L^{*}\right)$ and we note that $K_{1}^{e}$ is the number of distinct solutions $\boldsymbol{u}$ modulo $e$ of (7.2) for which G.C.D. $(\boldsymbol{u}, e)=1$. However $N_{e}(L *)$ is the number of solutions $\boldsymbol{x}$ modulo $e$ such that $e_{i} x_{i} \equiv 0(\bmod e)(i=1, \cdots$, $n)$. Clearly $N_{e}\left(L^{*}\right)=\prod_{i=1}^{n} e_{i}$. By Corollary 2 


$$
\mathfrak{R}=e^{n} \prod_{p \mid e, N\left(p, L^{\prime}\right)>0}\left(1-\frac{1}{p^{r(p, L)}}\right)
$$

where

$$
L^{\prime}=\left(\begin{array}{ccc}
e_{1} & & h_{1} \\
\bullet & & \vdots \\
& e_{n} & h_{n}
\end{array}\right) .
$$

Now $N\left(p, L^{\prime}\right)>0$ if and only if the system $e_{i} w_{i}+h_{i} \equiv 0(\bmod p)(i=$ $1, \cdots, n)$ is solvable, that is, if and only if G.C.D. $\left(p, e_{i}\right) \mid h_{i}$ or if and only if $p \nmid e_{i}$ or $p \mid$ G.C.D $\left(e_{i}, h_{i}\right)(i=1, \cdots, n)$. Also $r(p, L)$ is just the number of the $e_{i}(i=1, \cdots, n)$ not divisible by $p$. This completes the proof.

We now obtain a generalization of Steven's result [6] (see Corollary 3).

COROLlary 10. The equation

$$
\text { G.C.D. }\left(a_{1} x_{1}+b_{1}, \cdots, a_{n} x_{n}+b_{n}, c\right)=d,
$$

where

$$
\text { G.C.D. }\left(a_{1}, \cdots, a_{n}, d\right)=1 \text {, }
$$

is solvable if and only if

$$
\begin{aligned}
& d \mid c \text {, G.C.D. }\left(a_{i}, d\right) \mid b_{i}(i=1, \cdots, n), \\
& \text { G.C.D. }\left(a_{1}, \cdots, a_{n}, b_{1}, \cdots, b_{n}, c\right)=1 .
\end{aligned}
$$

The number of solution modulo $c$ is given by

$$
\prod_{i=1}^{n} \text { G.C.D. }\left(a_{i}, d\right) \cdot(c / d)^{n} \cdot \prod_{p \mid c / d}\left(1-\frac{\nu_{1}(p) \cdots \nu_{n}(p)}{p^{n}}\right),
$$

where $\nu_{i}(p)(i=1, \cdots, n)$ is the number of incongruent solutions modulo $p$ of $\frac{a_{i}}{\text { G.C.D. }\left(a_{i}, d\right)} x+\frac{b_{i}}{\text { G.C.D. }\left(a_{i}, d\right)} \equiv 0(\bmod p)$.

Proof. The necessary and sufficient conditions for solvability are immediate from Theorem 1 . When solvable we calculate the number $\mathfrak{N}$ of solutions modulo $c$ using Theorem 5 . Thus we require the number of distinct $\boldsymbol{u}$ modulo $e$ with G.C.D. $(\boldsymbol{u}, e)=1$ such that

$$
a_{i} x_{i}+b_{i} \equiv d u_{i}(\bmod d e)(i=1, \cdots, n)
$$

is solvable, that is, 


$$
\left(a_{i} / d_{i}\right) x_{i}+\left(b_{i} / d_{i}\right) \equiv\left(d / d_{i}\right) u_{i}\left(\bmod d / d_{i} \cdot e\right)
$$

where $d_{i}=$ G.C.D $\left(a_{i}, d\right)(i=1, \cdots, n)$.

This is solvable if and only if

$$
\text { G.C.D. }\left(\left(a_{i} / d_{i}\right),\left(d / d_{i}\right) e\right) \mid\left(d / d_{i}\right) u_{i}-\left(b_{i} / d_{i}\right)(i=1, \cdots, n),
$$

that is, if and only if,

$$
\left(d / d_{i}\right) u_{i} \equiv\left(b_{i} / d_{i}\right)\left(\bmod \text { G.C.D. }\left(\left(a_{i} / d_{i}\right), e\right)(i=1, \cdots, n) .\right.
$$

This system is equivalent to

$$
u_{i} \equiv h_{i}\left(\bmod \text { G.C.D. }\left(a_{i} / d_{i}, e\right)\right)(i=1, \cdots, n),
$$

where $h_{i}=\left(d / d_{i}\right)^{-1} b_{i} / d_{i}$ and $\left(d / d_{i}\right)^{-1}$ is an inverse of $d / d_{i}$ modulo G.C.D. $\left(a_{i} / d_{i}, e\right)$ since G.C.D. $\left(d / d_{i}, a_{i} / d_{i}, e\right)=1$. Thus by Corollary 9 the number of such $\boldsymbol{u}$ is

$$
\prod_{i=1}^{n} \frac{e}{\text { G.C.D. }\left(\left(a_{i} / d_{i}\right), e\right)} \prod_{p \mid e}^{\prime}\left(1-\frac{1}{p^{r(p)}}\right),
$$

where the dash $\left(^{\prime}\right)$ means that the product is taken over those $p \mid e$ such that $p \mid a_{i} / d_{i}$ or $p \mid$ G.C.D. $\left(a_{i} / d_{i}, b_{i} / d_{i}\right), i=1, \cdots, n$, as $p \mid$ G.C.D. $\left(a_{i} / d_{i}, e, h_{i}\right)$ if and only if $p \mid$ G.C.D. $\left(a_{i} / d_{i}, e, b_{i} / d_{i}\right)$ because $\left(d / d_{i}\right) h_{i} \equiv$ $b_{i} / d_{i}\left(\bmod\right.$ G.C.D. $\left(a_{i} / d_{i}, e\right)$ and G.C.D. $\left(d / d_{i}, a_{i} / d_{i}\right)=1(i=1, \cdots, n)$. Also $r(p)$ is the number of $a_{i} / d_{i}(i=1, \cdots, n)$ not divisible by $p$.

Next we need the number of incongruent $\boldsymbol{x}$ modulo de such that

$$
a_{i} x_{i} \equiv 0(\bmod d e)(i=1, \cdots, n) .
$$

This is just

$$
\begin{aligned}
& \prod_{i=1}^{n} \text { G.C.D. }\left(a_{i}, d e\right) \\
= & \prod_{i=1}^{n} d_{i} \text { G.C.D. }\left(a_{i} / d_{i},\left(d / d_{i}\right) e\right) \\
= & \prod_{i=1}^{n} d_{i} \text { G.C.D. }\left(a_{i} / d_{i}, e\right) .
\end{aligned}
$$

Hence by Theorem 5 the required number of solutions is

$$
\prod_{i=1}^{n}\left(d_{i} e\right) . \prod_{p \mid e}^{\prime}\left(1-\frac{1}{p^{r(p)}}\right),
$$

where the dash $\left({ }^{\prime}\right)$ means that the product is taken over those $p \mid e$ such that $p \mid a_{i} / d_{i}$ or $p \mid$ G.C.D. $\left(a_{i} / d_{i}, b_{i} / d_{i}\right), i=1, \cdots, n$. This number is

$$
\prod_{i=1}^{n} d_{i} \cdot e^{n} \cdot \prod_{\left.p\right|_{e}}\left(1-\frac{\nu_{1}(p) \cdots \nu_{n}(p)}{p^{n}}\right),
$$


as

$$
\nu_{i}(p)=\left\{\begin{array}{l}
1, p \nmid a_{i} / d_{i}, \\
0, p \mid a_{i} / d_{i}, p \nmid b_{i} / d_{i}, \\
p, p\left|a_{i} / d_{i}, p\right| b_{i} / d_{i} .
\end{array}\right.
$$

Finally we state that all formulas are easily modified if we do not assume $g=$ G.C.D. $\left(l_{1}, \cdots, l_{m}, d\right)=1$ (See introduction, Theorem 1 ). For example we list

Theorem 2'. If $\mathscr{S}_{d}^{c} \neq \varnothing$ the minimum modulus $M_{d}^{c}$ with respect to (1.1) is given by

$$
M_{d}^{c}=d_{1} \prod_{p \mid e, N\left(p d_{1}, L^{\prime} \mid g\right)>0} p
$$

Corollary 4'. If G.C.D. $(d, e)=1$ then the number $\mathfrak{N}_{d}^{c}$ of solutions of (1.1) modulo $M_{d}^{c}$ is

$$
\mathfrak{N}_{d}^{c}=N\left(d, L^{\prime} / g\right) \prod_{p ! e, N\left(p d_{1}, L^{\prime} / g\right)>0} p^{n}\left(1-\frac{1}{p^{r(p, L / g)}}\right) .
$$

\section{REFERENCES}

1. T. M. Apostol, Euler's $\varnothing$-function and separable Gauss sums, Proc. Amer. Math. Soc., 24 (1970), 482-485.

2. L. E. Dickson, History of the Theory of Numbers, Chelsea N.Y., (1952), 88-93.

3. D. N. Lehmer, Certain theorems in the theory of quadratic residues, Amer Math. Monthly, 20 (1913), 155-156.

4. H. J. S. Smith, On systems of linear indeterminate equations and congruences, Phil. Trans. Lond., 151 (1861), 293-326. (Collected Mathematical Papers Vol. 1, Chelsea N. Y. (1965), 367-409.)

5. R. Spira, Elementary problem no. E1730, Amer. Math. Monthly, 72 (1965), 907.

6. H. Stevens, Generalizations of the Euler $\varnothing$ - function, Duke Math. J., 38 (1971), 181-186.

Received November 30, 1970, and in revised form April, 1971. This research was supported by a National Research Council of Canada Grant (No. A-7233).

CARLETON UNiversity 


\section{PACIFIC JOURNAL OF MATHEMATICS}

\section{EDITORS}

H. SAMELSON

Stanford University

Stanford, California 94305

C. R. HobBY

University of Washington

Seattle, Washington 98105
J. DugundjI

Department of Mathematics

University of Southern California

Los Angeles, California 90007

RICHARD ARENS

University of California

Los Angeles, California 90024

\section{ASSOCIATE EDITORS}

E. F. BECKENBACH

B. H. NeUMaNN

F. WOLF

K. YoshidA

\section{SUPPORTING INSTITUTIONS}

UNIVERSITY OF BRITISH COLUMBIA

CALIFORNIA INSTITUTE OF TECHNOLOGY

UNIVERSITY OF CALIFORNIA

MONTANA STATE UNIVERSITY

UNIVERSITY OF NEVADA

NEW MEXICO STATE UNIVERSITY

OREGON STATE UNIVERSITY

UNIVERSITY OF OREGON

OSAKA UNIVERSITY
UNIVERSITY OF SOUTHERN CALIFORNIA STANFORD UNIVERSITY

UNIVERSITY OF TOKYO

UNIVERSITY OF UTAH

WASHINGTON STATE UNIVERSITY UNIVERSITY OF WASHINGTON

$\stackrel{*}{*} \stackrel{*}{*} \stackrel{*}{*}$ AMERICAN MATHEMATICAL SOCIETY
NAVAL WEAPONS CENTER 


\section{Pacific Journal of Mathematics}

Vol. 39 , No. 1

May, 1971

Charles A. Akemann, A Gelfand representation theory for $C^{*}$-algebras ....

Sorrell Berman, Spectral theory for a first-order symmetric system of

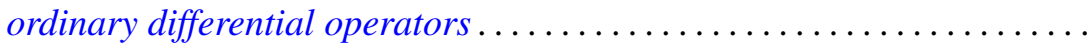

Robert L. Bernhardt, III, On splitting in hereditary torsion theories ........

J. L. Brenner, Geršgorin theorems, regularity theorems, and bounds for determinants of partitioned matrices. II. Some determinantal identities ..........................................

Robert Morgan Brooks, On representing $F^{*}$-algebras .............. 51

Lawrence Gerald Brown, Extensions of topological groups........... 71

Arnold Barry Calica, Reversible homeomorphisms of the real line ........ 79

J. T. Chambers and Shinnosuke Oharu, Semi-groups of local Lipschitzians in

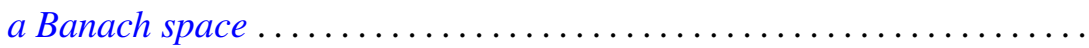

Thomas J. Cheatham, Finite dimensional torsion free rings .............

Byron C. Drachman and David Paul Kraines, A duality between

transpotence elements and Massey products ...................

Richard D. Duncan, Integral representation of excessive functions of a

Markov process ......................................

George A. Elliott, An extension of some results of Takesaki in the reduction

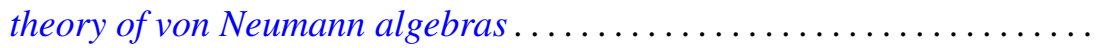

Peter C. Fishburn and Joel Spencer, Directed graphs as unions of partial

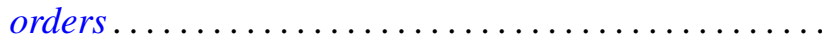

Howard Edwin Gorman, Zero divisors in differential rings ...

Maurice Heins, A note on the Löwner differential equations...

Louis Melvin Herman, Semi-orthogonality in Rickart rings. .

David Jacobson and Kenneth S. Williams, On the solution of linear G.C.D.

equations

Michael Joseph Kallaher, On rank 3 projective planes ... . .

Donald Paul Minassian, On solvable $O^{*}$-groups ...........

Nils Øvrelid, Generators of the maximal ideals of $A(\bar{D})$

Mohan S. Putcha and Julian Weissglass, A semilattice decomposition into

semigroups having at most one idempotent ............

Robert Raphael, Rings of quotients and $\pi$-regularity ....

J. A. Siddiqi, Infinite matrices summing every almost periodic sequence. .

Raymond Earl Smithson, Uniform convergence for multifunctions ...

Thomas Paul Whaley, Mulitplicity type and congruence relations in

universal algebras...

Roger Allen Wiegand, Globalization theorems for locally finitely generated modules... 